\title{
Differential Abnormal Pattern of Anterior Cingulate Gyrus Activation in Unipolar and Bipolar Depression: an fMRI and Pattern Classification Approach
}

\author{
Christian Bürger', Ronny Redlich', Dominik Grotegerd', Susanne Meinert', Katharina Dohm', \\ Ilona Schneider', Dario Zaremba', Katharina Förster', Judith Alferink ${ }^{1,2}$, Jens Bölte ${ }^{3}$, Walter Heindel ${ }^{4}$, \\ Harald Kugel ${ }^{4}$, Volker Arolt' ${ }^{\prime}$ and Udo Dannlowski ${ }^{*, 1,5}$ \\ 'Department of Psychiatry, University of Münster, Münster, Germany; ${ }^{2}$ Cluster of Excellence EXC 1003, Cells in Motion, University of Münster, \\ Münster, Germany; ${ }^{3}$ Institute of Psychology, University of Münster, Münster, Germany; ${ }^{4}$ Department of Clinical Radiology, University of Münster, \\ Münster, Germany; ${ }^{5}$ Department of Psychiatry, University of Marburg, Marburg, Germany
}

\begin{abstract}
Distinguishing bipolar disorder from major depressive disorder is a major challenge in psychiatric treatment. Consequently, there has been growing interest in identifying neuronal biomarkers of disorder-specific pathophysiological processes to differentiate affective disorders. Thirty-six depressed bipolar patients, 36 depressed unipolar patients, and 36 matched healthy controls (HCs) participated in an fMRI experiment. Emotional faces served as stimuli in a matching task. We investigated neural activation towards angry, fearful, and happy faces focusing on prototypical regions related to emotion processing, ie, the amygdala and the anterior cingulate gyrus (ACG). Furthermore, we employed a whole-brain and a multivariate pattern classification analysis. Unipolar patients showed abnormally reduced ACG activation toward happy and fearful faces compared with bipolar patients and HCs respectively. Furthermore, the whole-brain analysis revealed significantly increased activation in bipolar patients compared with unipolar patients in the fearful condition in the right frontal and parietal cortex. Moreover, the multivariate pattern classification analysis yielded significant classification rates of up to $72 \%$ based on ACG activation elicited by fearful faces. Our results question the rather 'amygdalocentric' neurobiological models of mood disorders. We observed patterns of abnormally reduced ventral and supragenual ACG activation, potentially indicating impaired bottom-up emotion processing and automatic emotion regulation specifically in unipolar but not in bipolar individuals.

Neuropsychopharmacology (2017) 42, 1399-|408; doi: I0.1038/npp.2017.36; published online I5 March 2017
\end{abstract}

\section{INTRODUCTION}

Distinguishing bipolar disorder (BD) from unipolar depression (UD) in individuals with a current depressive episode is a major issue in psychiatry with $60-70 \%$ of bipolar individuals being misdiagnosed as UD (Hirschfeld et al, 2003), frequently resulting in inadequate treatment (Bowden, 2010; Diler et al, 2013). Consequently, there has been growing interest in identifying neuronal biomarkers to distinguish affective disorders (eg, Almeida et al, 2009; Bertocci et al, 2012; Redlich et al, 2015).

In UD, studies repeatedly found a mood-congruent emotion processing bias in subcortical regions, notably the amygdala, ie, hypoactivation elicited by positive stimuli and hyperactivation in response to negative stimuli (eg, Stuhrmann et al, 2013; Suslow et al, 2010; for a review, see Stuhrmann et al, 2011).

\footnotetext{
*Correspondence: Professor $\cup$ Dannlowski, Department of Psychiatry, University of Münster, Albert-Schweitzer-Campus I, A9, Münster 48|49, Germany, Tel: +49 25। 835 66l0, Fax: +49 25I 8356612 , E-mail: dannlow@uni-muenster.de

Received 21 August 2016; revised 31 January 20 17; accepted 31 January 2017; accepted article preview online 16 February 2017
}

Moreover, studies indicate that the anterior cingulate gyrus (ACG) plays a major role in the pathophysiology of mood disorders (Phillips et al, 2003; 2008a; Strakowski et al, 2012). In their review Konarski et al (2008) found significant grey matter volume reductions in the ACG for BD and UD patients when compared with healthy controls (HC). Further, they report reduced amygdala volume in UD, whereas increased grey matter volume was found in BD individuals. In addition, activation patterns in the ACG, especially the subgenual ACG (sgACG), have been shown to successfully predict treatment response for antidepressant medication (eg, Mayberg, et al, 1997) and electroconvulsive therapy (Redlich et al, 2016). In a systematic review, Rive et al (2013) point out that the dorsal ACG (dACG), the rostral ACG (rACG), and the sgACG indicate a relative overrecruitment especially during automatic emotion control in UD.

For $\mathrm{BD}$, models hypothesize a general bottom-up hyperactivation (ie, increased automatic processing of emotionally salient stimuli and generation of emotional states) reflected by increased neural activation in limbic structures. On the contrary, top-down emotion regulation processes (ie, automatic and voluntary regulation, and reappraisal of 
emotional states) are assumed to be impaired, represented by reduced neural activation primarily in prefrontal structures (eg, Phillips et al, 2003; 2008a; Strakowski et al, 2005). However, a recent meta-analysis indicated heterogeneous findings of subcortical activation in $\mathrm{BD}$ compared with $\mathrm{HC}$ (Houenou et al, 2011). On the contrary, summarizing 50 whole-brain-based fMRI studies Chen et al (2011) found abnormally increased activation in $\mathrm{BD}$ patients relative to $\mathrm{HC}$ in the parahippocampus, hippocampus, and the amygdala. Overall, most fMRI studies comparing $\mathrm{BD}$ patients with HC were conducted in manic or remitted/ euthymic patients, whereas studies investigating depressed $\mathrm{BD}$ individuals are sparse.

Findings directly comparing $\mathrm{UD}, \mathrm{BD}$, and $\mathrm{HC}$ are rare (cf. Almeida and Phillips, 2013). In the majority of studies contrasting $\mathrm{UD}$ and $\mathrm{BD}$, a significant group $\times$ emotion interaction has been found primarily in the amygdale, ie, hyperactivation to negative stimuli in UD, whereas activation to positive stimuli appears rather heterogeneous with a trend for higher activation for $\mathrm{BD}$ individuals compared with UD

Table I Demographic and Clinical Characteristics of Participants

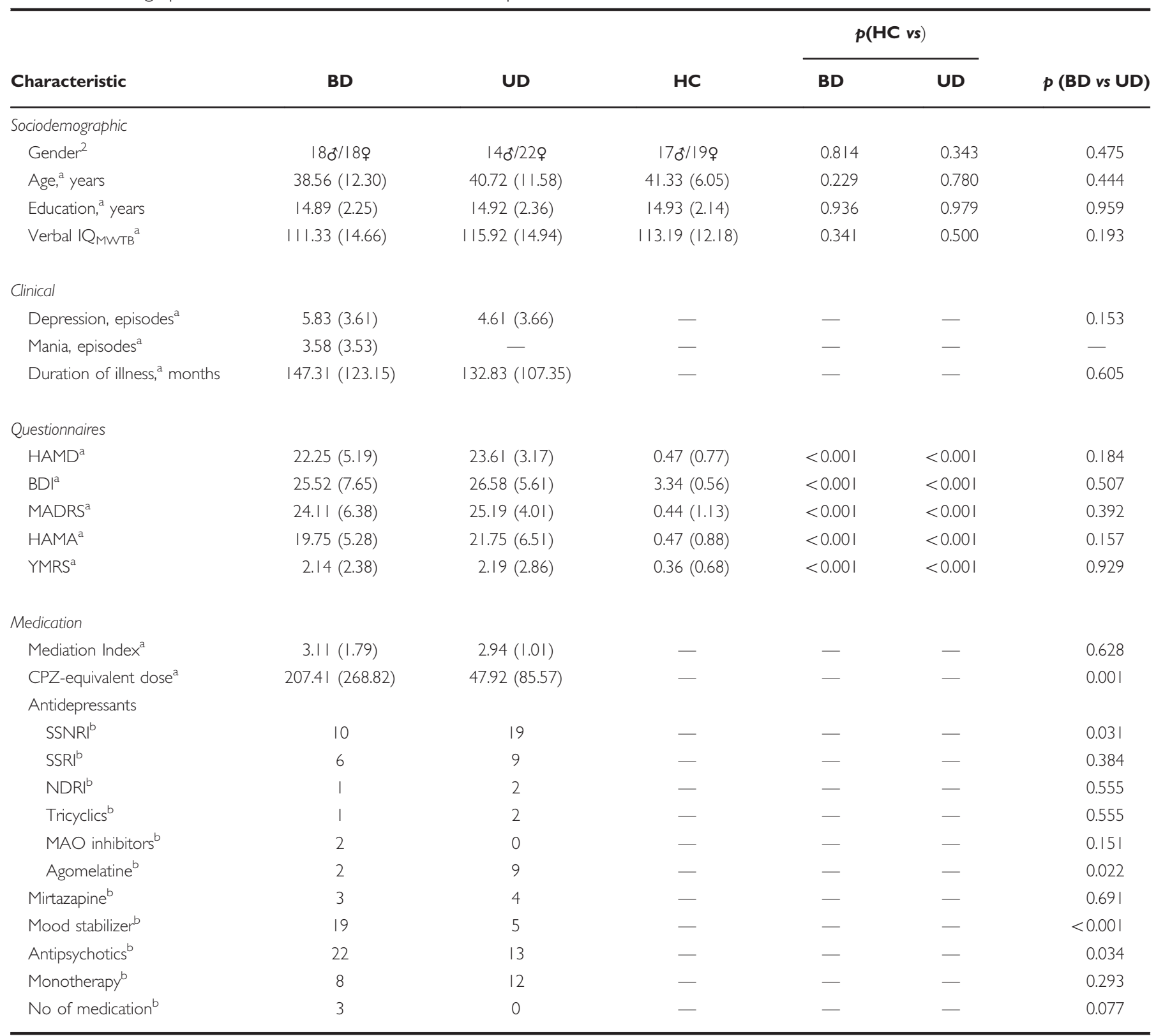

Abbreviations: BD, bipolar disorder, BDI, Beck Depression Inventory (Beck and Steer, 1987); CPZ, chlorpromazine; HAMA, Hamilton Anxiety Rating Scale (Hamilton, 1969); HAMD, Hamilton Depression Rating Scale (Hamilton, 1960); HC, healthy controls; MAO, monoamine oxidase; MWTB, Mehrfachwortwahl test (Lehrl, 2005); NDRI, norepinephrine-dopamine reuptake inhibitor, SSNRI, selective serotonin-norepinephrine reuptake inhibitor, SSRI, selective serotonin reuptake inhibitor; UD, unipolar disorder; YMRS, Young Mania Rating Scale (Young et al, 1978).

${ }^{a}$ Note: $t$-test (two-tailed).

${ }^{b} \chi^{2}$-test (two-tailed). 
(eg, Surguladze et al, 2010; Grotegerd et al, 2013; Lawrence et al, 2004). However, Almeida et al (2010) reported reduced amygdala reactivity in depressed $\mathrm{BD}$ patients compared with remitted $\mathrm{BD}$, depressed $\mathrm{UD}$ patients and $\mathrm{HC}$. In a metaanalysis directly comparing UD and BD individuals during facial affect processing tasks, Delvecchino et al (2012) found a general overactivation of limbic regions irrespectively of stimuli valence. They further observed increased activation in $\mathrm{BD}$ patients in the parahippocampal gyrus (including the amygdala), the bilateral ventral ACG, and the left pulvinar compared with UD patients, whereas UD patients showed increased activation patterns in the dACG.

Multivariate pattern classification studies reported reduced hippocampus and the amygdala volume in $\mathrm{BD}$ and reduced ACG volume in UD (Redlich et al, 2014). Furthermore, studies successfully classified UD patients based on amygdala hyperactivation toward negative stimuli, while hyperactivation toward positive stimuli was characteristic for BD patients (Grotegerd et al, 2014). Moreover, increased resting-state activity in the sgACG has been shown to successfully differentiate BD from UD patients (Almeida et al, 2013).

We directly compared depressed UD patients, depressed $\mathrm{BD}$ patients, and $\mathrm{HCs}$ in subcortical activation patterns in response to emotional faces during an fMRI scan. The amygdala and the ACG were investigated, because they are considered to be prototypical regions of emotion perception, especially automatic emotion processing. We hypothesized a mood-congruent processing bias in UD with a hypoactivation to happy faces and a hyperactivation to negative faces, whereas for $\mathrm{BD}$ patients we hypothesized a hyperactivation to both happy and negative faces. Finally, to add to the rather limited literature we employed a whole-brain analysis. Ultimately, we also employed a multivariate pattern classification approach in order to distinguish UD and BD based on neural activation.

\section{MATERIALS AND METHODS}

\section{Participants}

Thirty-six depressed bipolar patients (BD), 36 depressed unipolar patients (UD), as well as $36 \mathrm{HCs}$ participated in this study. Patients were inpatients recruited from the Department of Psychiatry of the University Hospital Münster. The clinical diagnosis in patients as well as the absence of past or current psychiatric disorders in $\mathrm{HC}$ was assessed using the SCID-I interview (Wittchen et al, 1997). Exclusion criteria for all participants were any neurological abnormalities, history of seizures, head trauma or substance dependency, current intake of benzodiazepines, as well as usual MRI exclusion criteria. The groups were carefully matched for sociodemographic characteristics (see Table 1). Patient groups did not differ in measurements of current psychopathology, in clinical variables of depressive illness history (see Table 1), and frequency of comorbid psychiatric disorders (see Supplementary Table S1).

To measure total medication load, we used a strategy as described in Redlich et al (2014). Each psychotropic medication was coded as absent $=0$, low $=1$ (equal or lower than average dose), or high $=2$ (greater than average dose), relative to the midpoint of the daily dose range recommended by Physician's Desk Reference. We calculated a composite measure of total medication load for each individual, reflecting dose and variety of different medications taken, by summing all individual medication (MEDIndex, cf. Redlich et al (2015)). Furthermore, to account for naturally higher intake of antipsychotic medication in $\mathrm{BD}$ patients, we converted individual antipsychotics into chlorpromazine equivalent dose (CPZ, cf. Woods (2003)).

Overall, BD and UD did not differ in medication load, whereas bipolar patients more frequently took mood stabilizers and antipsychotics. In contrast, the proportion of patients taking selective serotonin-noradrenalin reuptake inhibitor was higher in UD than in $\mathrm{BD}$, as well as the proportion of patients taking Agomelatine (see Table 1).

The study protocol was approved by the Ethics Committee of the University of Münster. Participants gave their full written informed consent and received a financial compensation.

\section{fMRI Paradigm}

A robust paradigm for eliciting subcortical, especially amygdala, activation using emotional faces that has been used in several studies was adapted and applied (Hariri et al, 2002; Dannlowski et al, 2012). The paradigm consisted of four blocks of a face-matching task and five blocks of a sensorimotor control task. Trials consisted of triplets of emotional faces (happy, angry, and fearful) taken from the NimStim database (Tottenham et al, 2009) or shapes (circles or ellipses). In each triplet, a reference image was presented in the upper part of the display. The same picture (target) and a face of a different person with the same emotional expression (distractor) were simultaneously presented side by side in the lower part of the display (see Supplementary Figure S1). Participants were asked to perform a basic matching task of faces and shapes, ie, indicating whether the left or right stimuli matches the reference stimuli above. Each block consisted of 12 trials, emotional faces were balanced block-wise for gender and emotional expression. Thus, within each face-block, all emotion conditions appeared, balanced for emotion in a pseudo-randomized order. Faces were presented for $2000 \mathrm{~ms}$ with a jittered inter-stimulusinterval (ISI) of $1000-5000 \mathrm{~ms}$ (mean $=3000 \mathrm{~ms}$ ), shapes were presented for $2000 \mathrm{~ms}$ with a fixed ISI of $1000 \mathrm{~ms}$.

\section{Image Acquisition}

Functional $\mathrm{T}^{*}$ weighted scans were acquired at a $3 \mathrm{~T}$ scanner (Gyroscan Intera 3T, Philips Medical Systems, Best, NL) using a single shot echoplanar sequence with parameters selected to minimize distortion in the central region of interest, while retaining adequate signal to noise ratio and $\mathrm{T} 2{ }^{*}$ sensitivity. Volumes consisting of 34 slices were acquired (matrix $64 \times 64$, resolution $3.6 \times 3.6 \times 3.6 \mathrm{~mm}$; $\mathrm{TR}=2.1 \mathrm{~s}$, $\mathrm{TE}=30 \mathrm{~ms}, \mathrm{FA}=90^{\circ}$ ). The slices were tilted $25^{\circ}$ from the AC/PC line to minimize drop-out artifacts in the orbitofrontal and mediotemporal region.

The paradigm presentation was projected to the rear end of the scanner (Sharp XG-PC10XE with additional HF shielding). During the experiment, participants lay supine in the MRI scanner with the response box in their right hand. 
The head position was stabilized with a vacuum head cushion.

\section{fMRI Data Analysis}

Functional scans were realigned and unwarped, normalized to a standard EPI-template reslicing with a resolution of
$2 \times 2 \times 2 \mathrm{~mm}$, and spatially smoothed with a Gaussian kernel of $6 \mathrm{~mm}$ (FWHM) using SPM8 (Wellcome Department of Cognitive Neurology, London, UK). For each subject, three contrast images were generated in the first-level analysis (happy $>$ shapes, angry $>$ shapes, and fearful > shapes) using an event-related analysis with a canonical hemodynamic response function. No subject had

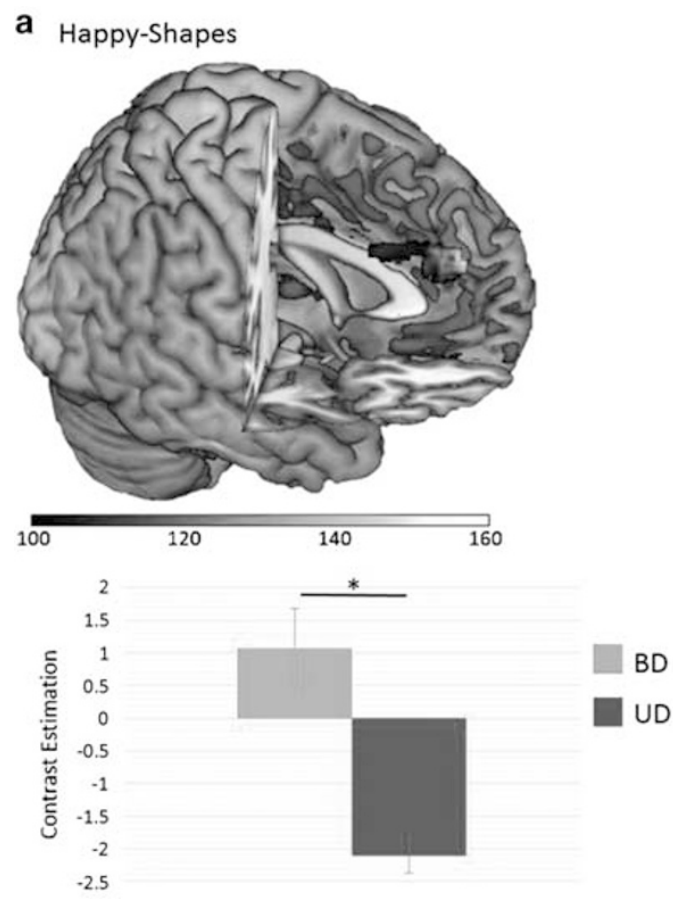

c
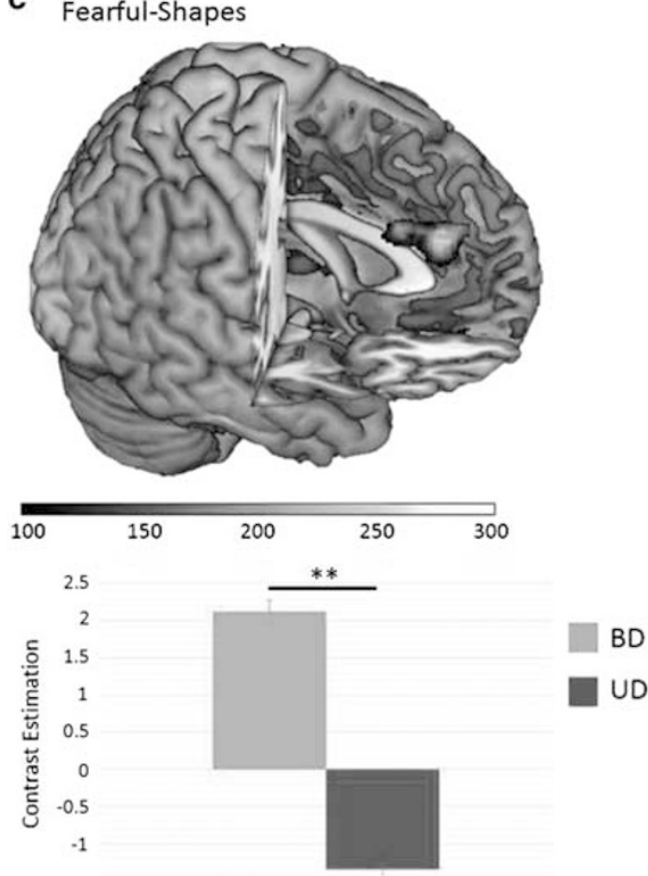

b Happy-Shapes
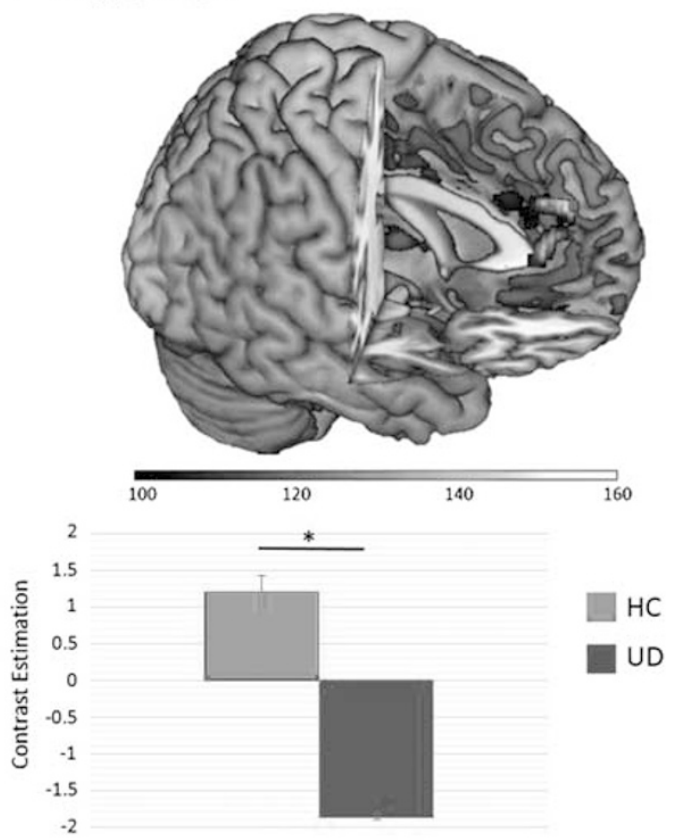

d Fearful-Shapes
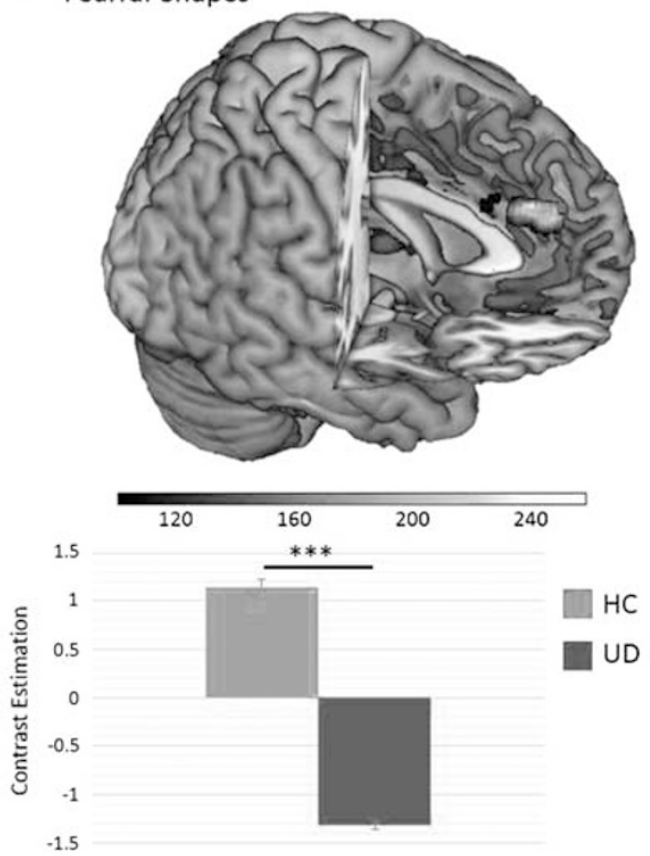

Figure I (a) ACG activation in the happy-shapes condition contrasting BD and UD patients. (b) ACG activation in the happy-shapes condition contrasting UD patients and HC. (c) ACG activation in the fearful-shapes condition contrasting BD and UD patients. (d) ACG activation in the fearful-shapes condition contrasting UD patients and HC. The bars depicting the estimated mean contrast values extracted from the significant clusters. Color bar: TFCE Score. $* p<0.05$ (TFCE $>100)$, *** $p<0.0$ I (TFCE $>170$ ), **** $p<0.00$ I (TFCE $>260$ ). Error bars indicate 95\% confidence intervals. ACG, anterior cingulate gyrus; $\mathrm{BD}$, bipolar disorder; HC, healthy controls; TFCE, threshold-free cluster enhancement; UD, unipolar disorder. A full color version of this figure is available at the Neuropsychopharmacology journal online. 

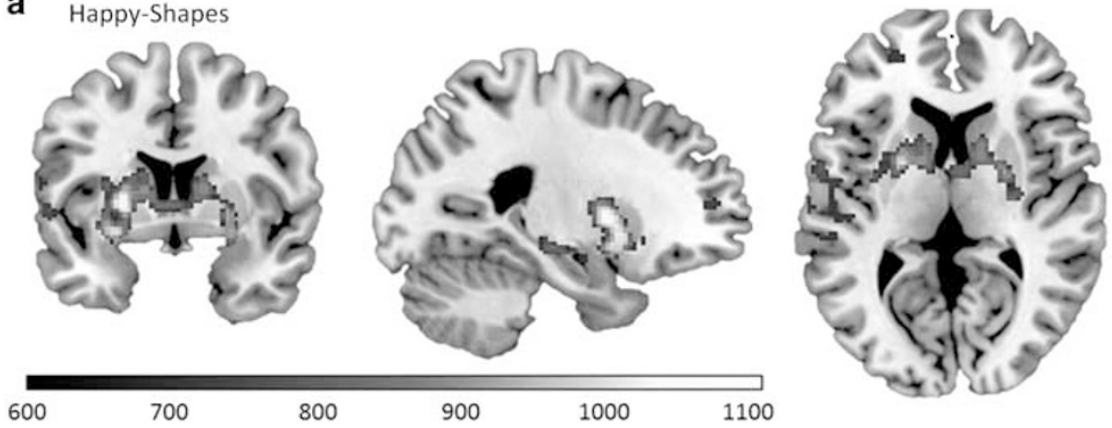

b Fearful-Shapes
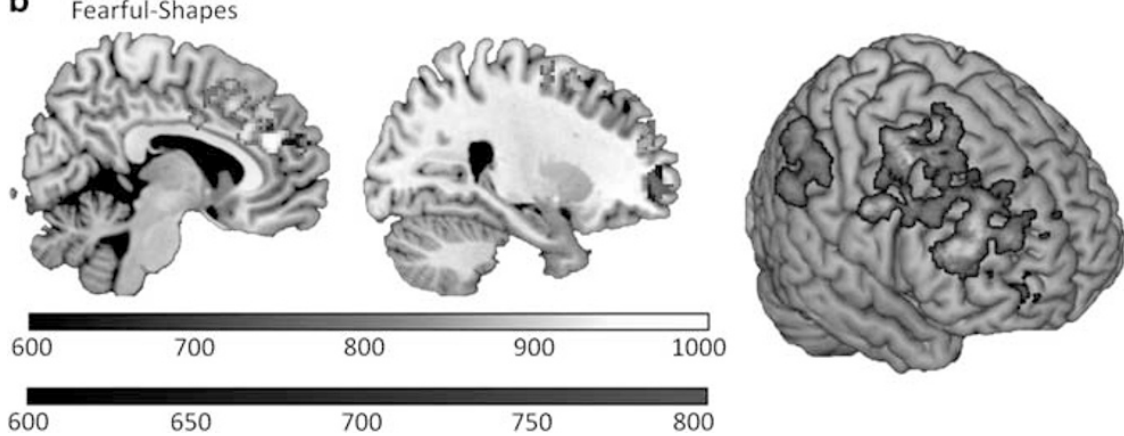

Figure 2 (a) Whole-brain activation in the happy-shapes condition contrasting UD patients and HC. Color bar: TFCE Score, indicating increased activation in UD patients compared with HC. (b) Whole-brain activation in the fearful condition contrasting BD and UD patients as well as UD patients and HC. Red/ yellow color bar depicts TFCE values of increased activation in BD patients compared with UD patients, Blue color bar depicts TFCE values of reduced activation in UD patients compared with HC. BD, bipolar disorder; HC, healthy controls; TFCE, threshold-free cluster enhancement; UD, unipolar disorder. A full color version of this figure is available at the Neuropsychopharmacology journal online.

to be excluded from the analysis due to excessive head movement $\left(>2 \mathrm{~mm}\right.$ or $\left.>2^{\circ}\right)$.

On the group level (second level analysis), contrast images from the first level were used for $t$-tests contrasting groups and emotional conditions.

In the second-level analysis, regions of interest (ROIs) had been defined a priori as prototypical structures for emotion processing: the amygdala and the ACG. These ROIs were defined according to the AAL atlas (Tzourio-Mazoyer et al, 2002). The anatomical mask was created using the WFUPickAtlas (http://fmri.wfubmc.edu/software/PickAtlas). Significance thresholds for multiple testing for the combined mask (2713 voxels in the bilateral ACG +468 voxels in the bilateral amygdala $=3181$ voxel) were obtained at the clusterlevel using threshold-free cluster enhancement (TFCE) as a non-parametric approach as implemented in the TFCEtoolbox (http://dbm.neuro.uni-jena.de/tfce, Version 93). We established a conservative FWE-corrected threshold for the ROI as well as for the whole-brain analysis obtained by 5000 permutations per test. We initially calculated two sample $t$-tests contrasting patients groups and HC, respectively, for each emotional condition. In order to inspect possible medication effects in the patient samples, we then entered the MED index and CPZ as covariates to the $t$-tests and compared the results. In addition, we calculated linear regressions for both medication indices. In a third step, we added clinical variables (BDI, HAMD, MARDS, YMRS, HAMA, number of depressive episodes, and duration of illness) in patient comparisons, compared results and calculated linear regressions for each variable. Finally, in order to inspect possible main effects of groups or emotional condition, we also conducted an exploratory 3 (group) $\times 3$ (emotion) repeated-measures ANOVA (see Supplementary Material).

\section{Multivariate Pattern Classification}

A multivariate pattern classification approach differentiating UD from BD patients was employed using the MANIA toolbox (https://bitbucket.org/grotegerd/mania, Grotegerd et al, 2014). All voxels of the bilateral amygdala and the bilateral ACG were separately extracted subject wise from the contrast images (happy $>$ shapes, angry $>$ shapes, and fearful $>$ shapes) and served as input for the pattern classification. Classification was calculated using liblinear Support Vector Machines and Gaussian Process Classifiers as described in Grotegerd et al (2014). Classifier performance was evaluated by using a leave-one-out per-group crossvalidation yielding accuracy, sensitivity, and specificity referring to the correct classification of unipolar or bipolar depressive subjects as average measures of performance over all validation steps. Statistical significance of classification was empirically estimated by performing permutation tests employing 1000 iterations.

\section{RESULTS}

\section{Behavioral Data}

The results of accuracy rates and reaction times are provided in the Supplementary Material. 
Table 2 Results of Post-Hoc Whole-Brain Analyses Contrasting Emotional Face Conditions

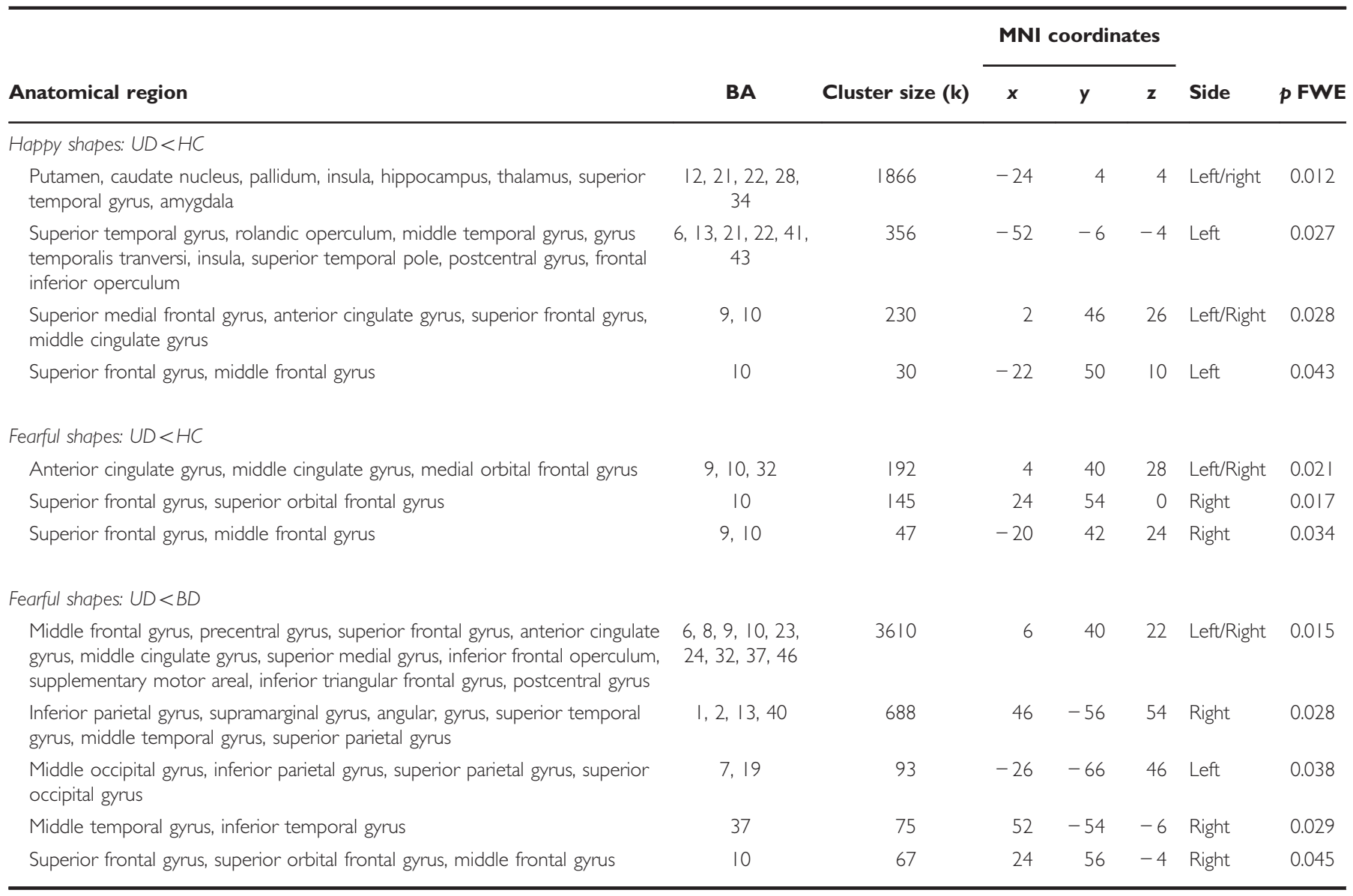

Abbreviations: BA, Brodmann areal; MNI, Montreal Neurological Institute, cluster $<30$ voxel not shown.

\section{Univariate Results}

In the happy condition, we found significantly reduced ACG activation in UD patients compared with BD patients (TFCE $(70)=159.29, k=154$ voxel, $p_{\text {corrected }}=0.015, x=4, y=50$, $z=24$; see Figure 1a) and HCs, respectively (TFCE $(70)=159.39, k=112, p_{\text {corrected }}=0.012, x=4, y=48, z=26$; see Figure $1 \mathrm{~b})$.

In the fearful condition we found significantly reduced right ACG activation in UD patients compared with $\mathrm{BD}$ patients $\left(\operatorname{TFCE}(70)=292.26, k=259\right.$ voxel, $p_{\text {corrected }}=0.001$, $x=6, y=40, z=22$; see Figure 1c) and HCs, respectively $\left(\mathrm{TFCE}_{1}(70)=257.65, k_{1}=149, p_{\text {corrected }}=0.001, x=4, y=40\right.$, $z=28 ; \operatorname{TFCE}_{1}(70)=110.77, k_{2}=16, p_{\text {corrected }}=0.038, x=4$, $y=26, z=26$ ); see Figure 1d).

Group differences in amygdala activation could neither be found in the happy nor the angry, nor the fearful condition. In addition, no ACG effects emerged in the angry condition.

The whole-brain analysis revealed reduced activation of UD patients compared with $\mathrm{HC}$ in the happy condition primarily in the basal ganglia, in frontal and temporal areas, the cingulate gyrus spreading to the amygdala, the insula, and the hippocampus (see Figure 2a and Table 2). Moreover, for BD compared with UD patients, we found significantly increased activation in the fearful condition primarily in the right frontal and parietal regions, and the cingulate gyrus. In addition, UD patients elicited reduced activation to fearful faces in the superior frontal gyrus and ACG, and middle cingulate gyrus compared with $\mathrm{HC}$ (see Figure $2 \mathrm{~b}$ and Table 2). Although the ROI analyses revealed ACG effects primarily in the supragenual part, due to additional statistical input from neighboring clusters, the whole brain analysis further yielded ACG effects in the right ventral part, revealing reduced activation to happy and fearful faces in UD patients compared with $\mathrm{HC}$ and reduced activation in UD patients compared with $\mathrm{HC}$ in a cluster projecting to the right perigenual part toward fearful faces (see Table 2 and Supplementary Figure S4).

Adding MED indices and $\mathrm{CPZ}$ as covariates did not significantly influence the results. Furthermore, there were no associations of medication indices with neural activation. Including clinical variables significantly reduced the wholebrain results of reduced activation in UD patients compared with $\mathrm{BD}$ patients toward fearful faces from primarily one extensive cluster and one moderate-sized cluster (see Table 2, 3610 and 688 voxel) to one significant cluster of 1162 voxel und 3 marginally significant clusters with moderate size (501 to 814 voxel) (see Supplementary Figure S2 and Supplementary Table S3). Regression analyses revealed that the reduction was solely driven by negative associations of neural activation with the HAMD scores $\left(p_{\max }=0.043\right.$, see Supplementary Figure S3), whereas there were no other 
significant associations with the other covariates. For the ROI analyses, only minor alterations emerged when adding clinical variables (see Supplementary Material).

Analyses of associations of ACG activation patterns with clinical variables revealed positive correlations of self-reported depressive symptoms (BDI) with ACG activation in the fearful condition (left: $\operatorname{TFCE}(61)=145.36, k=51$ voxel, $\left.p_{\text {corrected }}=0.025, x=-6, y=38, z=4\right)$. Moreover, negative associations emerged for ACG activation in the happy condition and clinicians depressions rating of the patients (HAMD, $\operatorname{TFCE}(61)=111.1, k=32$ voxel, $p_{\text {corrected }}=0.040, x=4, y=32$, $z=20$ ), for ACG activation in the angry condition and rated anxiety scores (HAMA, TFCE $(61)=157.35, k=98$ voxel, $\left.p_{\text {corrected }}=0.012, x=-2, y=36, z=14\right)$ and for ACG activation in the fearful condition and HAMD scores $(\operatorname{TFCE}(61)=168.49$, $k=192$ voxel, $p_{\text {corrected }}=0.014, x=-6, y=26, z=18$ ). For further information and scatterplots, see Supplementary Material.

The results of the exploratory 3 (group) $\times 3$ (emotion) ANOVA are provided in the Supplementary Material.

\section{Multivariate Pattern Classification Results}

Table 3 shows the results of the binary pattern classifications. With up to $72.22 \%$ accuracy (sensitivity $=80.56 \%$, specificity $=63.89 \%)$, the most significant results $(p=0.004)$ were achieved using the fearful > shapes contrast in the ACG. In addition, the happy $>$ shapes contrast yielded significant results for the amygdala (accuracy $=$ sensitivity $=$ specificity $=$ $63.89 \%, p=0.028$ ). Furthermore, classification based on ACG activation in the happy > shapes condition significantly distinguished UD patients from HC (accuracy = sensitivity = specificity $=66.67 \%, p=0.017$ ).

\section{DISCUSSION}

To the best of our knowledge, this is one of the largest studies investigating neural correlates of facial emotion processing in depressed bipolar patients, depressed unipolar patients, and HCs. Our results revealed abnormally reduced ACG activation in depressed UD compared with depressed BD patients and HC. Furthermore, the whole-brain analysis yielded abnormally reduced activation elicited by happy faces in the basal ganglia, in frontal and temporal areas, as well as in limbic structures, notably the cingulate gyrus spreading to the amygdala, the insula and the hippocampus. Moreover, UD patients exhibited significantly reduced neural activation compared with HC in the fearful condition in frontal areas and the ventral cingulate gyrus. In addition, for $\mathrm{BD}$ patients compared with UD patients, we found significantly increased activation in the fearful condition primarily in the frontal and parietal regions, and the dorsal cingulate gyrus. However, these whole-brain differences where partially reduced when controlling for current ratings of depressive symptoms. Besides, primarily negative associations of dACG activation and clinical ratings emerged. Finally, multivariate pattern classification results achieved significant above chance classification rates of up to $72 \%$ based on ACG activation elicited by happy and fearful faces, as well as based on amygdala activation toward happy faces.
Table 3 Binary Multivariate Pattern Classification of UD and BD Patients

\begin{tabular}{llll}
\hline & \multicolumn{3}{c}{ Accuracy (p-value) } \\
\cline { 2 - 4 } & BD vs UD & UD vs HC & BD vs HC \\
\hline Amygdala & & \\
$\quad$ Support vector machines & & \\
$\quad$ Happy > shapes & $59.72(0.102)$ & $52.78(0.371)$ & $59.72(0.109)$ \\
Fearful > shapes & $47.22(0.691)$ & $50.00(0.515)$ & $48.61(0.622)$ \\
Angry > shapes & $54.17(0.311)$ & $51.39(0.439)$ & $56.94(0.182)$ \\
Gaussian process classifier & & \\
$\quad$ Happy > shapes & $\mathbf{6 3 . 8 9}(\mathbf{0 . 0 2 8}) *$ & $54.17(0.325)$ & $58.33(0.137)$ \\
Fearful > shapes & $48.61(0.619)$ & $52.78(0.358)$ & $54.17(0.286)$ \\
Angry > shapes & $59.72(0.099)$ & $50.00(0.564)$ & $55.56(0.254)$
\end{tabular}

Anterior cingulate gyrus

Support vector machines

$\begin{array}{lcll}\text { Happy > shapes } & 62.50(0.059) & 61.11(0.081) & 48.61(0.567) \\ \text { Fearful > shapes } & \mathbf{6 9 . 4 4 ( \mathbf { 0 . 0 0 8 } ) * *} & 51.39(0.444) & 52.75(0.372) \\ \text { Angry > shapes } & 51.39(0.442) & 48.61(0.638) & 55.56(0.261)\end{array}$

Gaussian process classifier

Fearful > shapes $\quad \mathbf{7 2 . 2 2}(\mathbf{0 . 0 0 4})^{* * *} \quad 56.94(0.174) \quad 50.00(0.647)$

Angry > shapes $\quad 50.00(0.538) \quad 44.44(0.284) \quad 54.17(0.346)$

\begin{tabular}{|c|c|c|c|}
\hline \multicolumn{4}{|l|}{ Whole brain } \\
\hline \multicolumn{4}{|c|}{ Support vector machines } \\
\hline Happy > shapes & $50.00(0.505)$ & $61.11(0.086)$ & $41.67(0.117)$ \\
\hline Fearful > shapes & $54.17(0.308)$ & $55.56(0.267)$ & $44.44(0.257)$ \\
\hline Angry > shapes & $48.61(0.409)$ & $55.56(0.296)$ & $43.06(0.182)$ \\
\hline \multicolumn{4}{|c|}{ Gaussian process classifier } \\
\hline Happy > shapes & $50.00(0.643)$ & $62.50(0.074)$ & $50.00(0.621)$ \\
\hline Fearful > shapes & $58.33(0.162)$ & $58.33(0.129)$ & $43.06(0.201)$ \\
\hline Angry > shapes & $45.83(0.358)$ & $51.39(0.453)$ & $37.50(0.072)$ \\
\hline
\end{tabular}

Abbreviations: $\mathrm{BD}$, bipolar disorder; $\mathrm{HC}$, healthy controls; UD, unipolar depression.

$* p<0.05$, *** $p<0.01$. Accuracy in \%. Bold represents statistically significant results $(p<0.05)$.

Concerning the amygdala, we did not find differential patterns of activation between groups and facial emotion expression. Given the amount of fMRI literature about mood-congruent amygdala responses in UD patients, this finding appears surprising (cf. Stuhrmann et al, 2011). On closer examination of the cited review, abnormal hyperactivation to negative faces in UD is predominately found for sad faces while only 2 out of 20 studies reported an increased amygdala response to combined contrasts (fearful-sad, Peluso et al, 2009; fearful-angry, Zhong et al, 2011) and only study reported an increased amygdala response to fearful faces (Sheline et al, 2001). Furthermore, the literature of amygdala activation to happy faces in UD is inconclusive. Moreover, in their meta-analysis Delvecchio et al (2012) question 'the current amygdalocentric models for mood disorders' due to methodologic issues and task relevant considerations. 
Concerning the ACG, we found abnormally reduced activation in UD patients elicited by happy and fearful faces. Although the dACG and sgACG seem to be crucial for emotion regulation, the ventral and sgACG appear to be involved in the generation and recognition of emotional states (Bush et al, 2000). For UD patients, the literature indicates hypoactivation in the dACG and hyperactivation in the ventral ACG/sgACG (cf. Stuhrmann et al, 2011). The significant clusters of the ROI analyses are located in the supragenual ACG, ie, rather in the dorsal 'cognitive' subdivision of the ACG, whereas the whole-brain analysis further revealed clusters in the ventral 'affective' subdivision of the ACG. Reduced activation in the ventral part of the ACG could be interpreted as reduced bottom-up emotional processing, whereas reduced dACG activation could be interpreted as reduced top-down regulation of emotional processing (either automatic or voluntary emotion regulation, $c f$. Rive et al, 2013). Given the employed paradigm (supraliminal presentation of emotional faces with implicit emotion processing task), it can be speculated that reduced activation to happy and fearful faces in UD patients might indicate an impaired automatic rather than voluntary emotion regulation process. In addition, regression analyses indicated that increased depressive and anxiety symptoms were associated with reduced dACG activation, potentially suggesting reduced impaired regulatory processes. However, the absence of group $\times$ emotion interaction effects in the amygdala complicates the interpretation of the abnormal ACG hypoactivation.

In our study, depressed BD patients did not differ from HC. For BD patients, meta-analytic findings report aberrant reductions in the activation of the ACG to both happy and fearful faces when analyzing BD patients across all affective states (manic, depressed, and euthymic; Delvecchio et al, 2012). In contrast, when comparing only depressed $\mathrm{BD}$ patients with HC, Chen et al (2011) observed no significant differences during emotion perception in their meta-analysis, which is line with our results. One explanation could be that abnormal limbic activation patterns are highly state dependent, ie, hyperactivation in euthymic state might be downregulated by depressive symptoms and might therefore not be different from HC. However, further studies are needed to answer this question.

Multivariate pattern classification results yielded significant but rather small above chance classification rates based primarily on ACG activation towards fearful faces in UD patients complementing univariate findings. Given the issue of great heterogeneity in fMRI paradigms and resulting issues of practicability classification based on structural MRI or resting-state MRI might appear more promising as diagnostic markers for clinical practice with reported classification rates of about $80 \%$ (eg, Almeida et al, 2013; Redlich et al, 2014).

Taken together, our findings indicate reduced ventral and supragenual ACG activation in UD during an implicit emotion processing paradigm presenting facial expression at a supraliminal level of consciousness. Based on the anatomical subdivision of the ACG, we speculate that the observed patterns of hypoactivation in UD patients could be interpreted as reduced bottom-up emotion perception of both positive and negative stimuli, as well as impaired topdown emotion regulation. Nevertheless, the absence of amygdala effects remains not fully explicable and requires further investigation and replication. Interestingly, depressed bipolar individuals did not show similar regulatory impairments. Thus, it is conceivable that depressed unipolar patients experience reduced emotional reactivity to both positive and negative stimuli and therefore require reduced regulation. Clinically, the symptom of flattened affect might be the overt expression of this reduced emotional reactivity. To elaborate this hypothesis further, one could investigate remitted UD and BD patients as well as manic BD patients in future studies. Finally, the exploratory whole-brain analysis yielded additional regional differences between BD and UD patients primarily in the frontal and parietal region that should be examined in further studies.

\section{CONCLUSION}

In sum, we found abnormally reduced ACG activation to positive and negative stimuli specific for depressed unipolar but not for depressed bipolar individuals. The findings contribute to the pathophysiological understanding of mood disorders indicating state-dependency of neural activation pattern especially in BD. Finally, our findings question primarily 'amygdalocentric' models of mood disorders.

\section{Limitations}

First, all but three patients were medicated with differences in the distribution of medications between patient groups which complicate the interpretation of results. Albeit, overall the medication index did not differ between the patient's samples and correcting for medication intake did not significantly alter the effects. Furthermore, in recent fMRI studies no significant association of psychotropic medication and ACG has been reported ( $c f$. Phillips et al, 2008b). However, in the due to software limitations we did not correct for medication in the multivariate pattern classification. After all, findings should be replicated in studies with unmedicated patients.

Second, we investigated neural correlates of emotion processing in currently depressed unipolar and bipolar patients. Thus, our findings cannot be generalized to other mood states. Further studies should compare depressed as well as euthymic/ remitted $\mathrm{UD}$ and $\mathrm{BD}$ individuals and manic $\mathrm{BD}$ patients.

Third, due to limited availability of the sample, we calculated leave-one-out per-group cross-validation. It would be desirable to rather use leave-n-out cross-validation or independent training and test samples in further studies.

Finally, our facial paradigm did not include faces with a sad expression. As noted, findings about mood-congruent neural responses are rather heterogeneous, especially for negative faces. Subsequent work should aim to differentiate activation patterns elicited by specific negative facial expressions rather than subsuming different expression as 'negative faces'.

\section{FUNDING AND DISCLOSURE}

$\mathrm{V}$. Arolt is a member of the advisory board of, or has given presentations on behalf of, the following companies: AstraZeneca, Janssen-Organon, Lilly, Lundbeck, Servier, Pfizer, 
Otsuka, and Trommsdorff. These affiliations are of no relevance to the work described in the manuscript. H. Kugel has received consultation fees from MR:comp GmbH, Testing Services for MR Safety. This cooperation has no relevance to the work that is covered in the manuscript. The study was supported by grants of the German Research Foundation (DFG; grant FOR 2107; DA1151/5-1 to UD; grant SFB-TRR58, Project C09 to UD) and the IZKF of the medical faculty of Münster (grant Dan3/012/17 to UD). The authors declare no conflict of interest.

\section{REFERENCES}

Almeida JRC, Mourao-Miranda J, Aizenstein HJ, Versace A, Kozel FA, Lu H et al (2013). Pattern recognition analysis of anterior cingulate cortex blood flow to classify depression polarity. Br J Psychiatry 203: 310-311.

Almeida JRC, Phillips ML (2013). Distinguishing between unipolar depression and bipolar depression: current and future clinical and neuroimaging perspectives. Biol Psychiatry 73: 111-118.

Almeida JRC, Versace A, Hassel S, Kupfer DJ, Phillips ML (2010). Elevated amygdala activity to sad facial expressions: a state marker of bipolar but not unipolar depression. Biol Psychiatry 67: 414-421.

Almeida JRC, Versace A, Mechelli A, Hassel S, Quevedo K, Kupfer DJ et al (2009). Abnormal amygdala-prefrontal effective connectivity to happy faces differentiates bipolar from major depression. Biol Psychiatry 66: 451-459.

Beck AT, Steer RA (1987). Beck Depression Inventory: Manual. Psychological Corporation, Harcourt Brace Jovanovich: San Antonio, TX.

Bertocci MA., Bebko GM, Mullin BC, Langenecker SA, Ladouceur CD, Almeida JRC et al (2012). Abnormal anterior cingulate cortical activity during emotional n-back task performance distinguishes bipolar from unipolar depressed females. Psychol Med 42: 1417-1428.

Bowden CL (2010). Diagnosis, treatment, and recovery maintenance in bipolar depression. J Clin Psychiatry 71: 1-478.

Bush G, Luu P et al (2000). Cognitive and emotional influences in anterior cingulate cortex. Trends Cogn Sci 4: 215-222.

Chen CH, Suckling J, Lennox BR, Ooi C, Bullmore ET (2011). A quantitative meta-analysis of fMRI studies in bipolar disorder. Bipolar Disord 13: 1-15.

Dannlowski U, Stuhrmann A, Beutelmann V, Zwanzger P, Lenzen T, Grotegerd D et al (2012). Limbic scars: long-term consequences of childhood maltreatment revealed by functional and structural magnetic resonance imaging. Biol Psychiatry 71: 286-293.

Delvecchio G, Fossati P, Boyer P, Brambilla P, Falkai P, Gruber O et al (2012). Common and distinct neural correlates of emotional processing in Bipolar Disorder and Major Depressive Disorder: a voxel-based meta-analysis of functional magnetic resonance imaging studies. Eur Neuropsychopharmacol 22: 100-113.

Diler RS, de Almeida JRC, Ladouceur C, Birmaher B, Axelson D, Phillips ML (2013). Neural activity to intense positive versus negative stimuli can help differentiate bipolar disorder from unipolar major depressive disorder in depressed adolescents: a pilot fMRI study. Psychiatry Res 214: 277-284.

Grotegerd D, Redlich R, Almeida JR, Riemenschneider M, Kugel H, Arolt V et al (2014). MANIA-A pattern classification toolbox for neuroimaging data. Neuroinformatics 12: 471-486.

Grotegerd D, Stuhrmann A, Kugel H, Schmidt S, Redlich R, Zwanzger P et al (2014). Amygdala excitability to subliminally presented emotional faces distinguishes unipolar and bipolar depression: an $\mathrm{fMRI}$ and pattern classification study. Hum Brain Mapp 35: 2995-3007.
Grotegerd D, Suslow T, Bauer J, Ohrmann P, Arolt V, Stuhrmann A et al (2013). Discriminating unipolar and bipolar depression by means of fMRI and pattern classification: a pilot study. Eur Arch Psychiatry Clin Neurosci 263: 119-131.

Hamilton M (1960). A rating scale for depression. I Neurol Neurosurg 23: 56-63.

Hamilton M (1969). Diagnosis and rating of anxiety. Br J Psychiatry 3: 76-79.

Hariri AR, Mattay VS, Tessitore A, Kolachana B, Fera F, Goldman D et al (2002). Serotonin transporter genetic variation and the response of the human amygdala. Science 297: 400-403.

Hirschfeld RM, Lewis L, Vornik LA (2003). Perceptions and impact of bipolar disorder: how far have we really come? Results of the national depressive and manic-depressive association 2000 survey of individuals with bipolar disorder. J Clin Psychiatry 64: 161-174.

Houenou J, Frommberger J, Carde S, Glasbrenner M, Diener C, Leboyer M (2011). Neuroimaging-based markers of bipolar disorder: evidence from two meta-analyses. J Affect Disord 132: 344-355.

Konarski JZ, McIntyre RS, Kennedy SH, Rafi-Tari S, Soczynska JK, Ketter TA (2008). Volumetric neuroimaging investigations in mood disorders: Bipolar disorder versus major depressive disorder. Bipolar Disord 10: 1-37.

Lawrence NS, Williams AM, Surguladze S, Giampietro V, Brammer MJ, Andrew C et al (2004). Subcortical and ventral prefrontal cortical neural responses to facial expressions distinguish patients with bipolar disorder and major depression. Biol Psychiatry 55: 578-587.

Lehrl S (2005). Mehrfachwahl-Wortschatz-Intelligenztest MWT-B. Spitta Verlag: Balingen 2005.

Mayberg HS, Brannan SK, Mahurin RK, Jerabek PA, Brickman JS, Tekell JL et al (1997). Cingulate function in depression: a potential predictor of treatment response. Neuroreport 8: 1057-1061.

Peluso MA, Glahn DC, Matsuo K, Monkul ES, Najt P, Zamarripa F et al (2009). Amygdala hyperactivation in untreated depressed individuals. Psychiatry Res 173: 158-161.

Phillips ML, Drevets WC, Rauch SL, Lane R (2003). Neurobiology of emotion perception II: Implications for major psychiatric disorders. Biol Psychiatry 54: 515-528.

Phillips ML, Ladouceur CD, Drevets WC (2008a). A neural model of voluntary and automatic emotion regulation: implications for understanding the pathophysiology and neurodevelopment of bipolar disorder. Mol Psychiatry 13: 833-857.

Phillips ML, Travis MJ, Kupfer DJ, Fagiolini A (2008b). Medication effects in neuroimaging studies of bipolar disorder. $A m J$ Psychiatry 165: 313-320.

Redlich R, de Almeida JCR, Grotegerd D, Opel N, Kugel H, Heindel W et al (2014). Brain morphometric biomarkers distinguishing unipolar and bipolar depression: a Voxel-based morphometrypattern classification approach. JAMA Psychiatry 71: 1222-1230.

Redlich R, Dohm K, Grotegerd D, Opel N, Zwitserlood P, Heindel W et al (2015). Reward processing in unipolar and bipolar depression: a functional MRI study. Neuropsychopharmacology 40: 2623-2631.

Redlich R, Opel N, Grotegerd D, Dohm K, Zaremba D, Bürger C et al (2016). Prediction of individual response to electroconvulsive therapy via machine learning on structural magnetic resonance imaging data. JAMA Psychiatry 73: 557-564.

Rive MM, van Rooijen G, Veltman DJ, Phillips ML, Schene AH, Ruhé HG (2013). Neural correlates of dysfunctional emotion regulation in major depressive disorder. A systematic review of neuroimaging studies. Neurosci Biobehav Rev 37: 2529-2553.

Sheline YI, Barch DM, Donnelly JM, Ollinger JM, Snyder AZ, Mintun MA (2001). Increased amygdala response to masked emotional faces in depressed subjects resolves with antidepressant treatment: an fMRI study. Biol Psychiatry 50: 651-658.

Strakowski SM, Adler CM, de Almeida JCR, Altshuler LL, Blumberg HP, Chang KD et al (2012). The functional neuroanatomy of bipolar disorder: a consensus model. Bipolar Disord 14: 313-325. 
Strakowski SM, Delbello MP, Adler CM (2005). The functional neuroanatomy of bipolar disorder: a review of neuroimaging findings. Mol Psychiatry 10: 105-116.

Stuhrmann A, Dohm K, Kugel H, Zwanzger P, Redlich R, Grotegerd D et al (2013). Mood-congruent amygdala responses to subliminally presented facial expressions in major depression: associations with anhedonia. J Psychiatry Neurosci 38: 249-258.

Stuhrmann A, Suslow T, Dannlowski U (2011). Facial emotion processing in major depression: a systematic review of neuroimaging findings. Biol Mood Anxiety Disord 1: 1-10.

Surguladze SA, Marshall N, Schulze K, Hall MH, Walshe M, Bramon $\mathrm{E}$ et al (2010). Exaggerated neural response to emotional faces in patients with bipolar disorder and their first-degree relatives. Neuroimage 53: 58-64.

Suslow T, Konrad C, Kugel H, Rumstadt D, Zwitserlood P, Schöning $S$ et al (2010). Automatic mood-congruent amygdala responses to masked facial expressions in major depression. Biol Psychiatry 67: 155-160.
Tottenham N, Tanaka JW, Leon AC, McCarry T, Nurse M, Hare TA et al (2009). The NimStim set of facial expressions: judgments from untrained research participants. Psychiatry Res 168: $242-249$.

Tzourio-Mazoyer N, Landeau B, Papathanassiou D, Crivello F, Etard O, Delcroix N et al (2002). Neuroimage 15: 273-289.

Wittchen H-U, Wunderlich U, Gruschwitz S, Zaudig M (1997). Strukturiertes Klinisches Interview für DSM-IV. Hogrefe: Goettingen, 1997.

Woods SW (2003). Chlorpromazine equivalent doses for the newer atypical antipsychotics. J Clin Psychiatry 64: 663-667.

Young RC, Biggs JT, Ziegler VE, Meyer DA (1978). A rating scale for mania: reliability, validity and sensitivity. Br J Psychiatry 133: 429-435.

Zhong M, Wang X, Xiao J, Yi J, Zhu X, Liao J et al (2011). Amygdala hyperactivation and prefrontal hypoactivation in subjects with cognitive vulnerability to depression. Biol Psychiatry 88: $233-242$.

Supplementary Information accompanies the paper on the Neuropsychopharmacology website (http://www.nature.com/npp) 